\title{
Cronotipo e implicações para sua utilização na fisioterapia em pacientes com acidente vascular encefálico
}

\author{
Chronotype and implications for its use in physical therapy in patients with stroke
}

Tania Fernandes Campos $^{1}$, Carolina Dutra Gomes Pinheiro ${ }^{2,3}$, Fabíola Pimentel Diógenes ${ }^{3}$, Marina Tostes Miranda Barroso ${ }^{3}$, Ana Amália Torres Souza Gandour Dantas ${ }^{4}$

Estudo desenvolvido na Universidade Federal do Rio Grande do Norte, Hospital Universitário Onofre Lopes, Departamento de Fisioterapia, Laboratório de Cronobiologia e de Movimento em Saúde.

1 Doutora em Psicobiologia; Professora de Fisioterapia Neurológica do Departamento de Fisioterapia da Universidade Federal do Rio Grande do Norte (UFRN) - Natal (RN), Brasil.

2 Especialista em RPG/ Reposturar-se.

${ }^{3}$ Fisioterapeuta.

${ }^{4}$ Mestre em Psicologia Clínica; Psicóloga.

\section{ENDERECO PARA}

\section{CORRESPONDÊNCIA}

Tania Fernandes Campos Departamento de Fisioterapia da UFRN - Av. Senador Salgado Filho, 3.000 - CEP: 59066-800 - Natal (RN), Brasil - E-mail: taniacampos@ufrnet.br

\section{APRESENTAÇÃO} novembro/2010

\section{ACEITO PARA PUBLICAÇÃO} julho/2011

FONTE DE FINANCIAMENTO nenhuma

CONFLITO DE INTERESSES nada a declarar

\section{APRESENTAÇÃO EM EVENTO} CIENTÍFICO: \section{Congresso de Iniciação} Científica da UFRN.

Parecer de aprovação no Comitê de Ética no 164/06.
RESUMO: Na prática clínica, frequentemente o fisioterapeuta avalia e programa o tratamento do paciente sem levar em consideração a variação temporal de funções e comportamentos. O objetivo do estudo foi analisar a influência do cronotipo, padrão vigília-sono (qualidade do sono e sonolência excessiva) e regularidade do estilo de vida na determinação do horário de preferência para a prática de atividades física e mental em pacientes com acidente vascular encefálico (AVE). Participaram

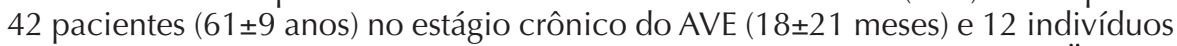

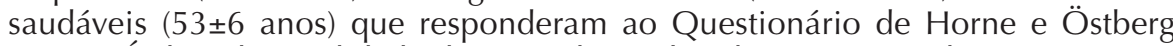
(QHO), Índice de Qualidade do Sono de Pittsburgh (IQSP), Sonolência Excessiva de Epworth (SEE) e o Social Rhythm Metric (SRM). Foi questionado em qual horário os participantes preferiam realizar atividade física (exercícios) e mental (tarefas de raciocínio), considerando apenas seu bem-estar pessoal. Os dados foram analisados através do teste do $\chi^{2}$ e regressão múltipla. Dos pacientes avaliados, $93 \%$ eram matutinos, $64 \%$ apresentavam qualidade ruim do sono, $43 \%$ sonolência excessiva e $57 \%$ padrão irregular da rotina diária. Ao final da análise de regressão verificou-se que o cronotipo foi o único fator que teve influência no horário preferencial das atividades. Esses achados sugerem a necessidade da avaliação do cronotipo dos pacientes antes de se estabelecer um horário de atendimento na Fisioterapia.

Descritores: acidente cerebral vascular; transtornos do sono; estilo de vida; ritmo circadiano.

ABSTRACT: In clinical practice, the physical therapist often evaluates and programs the patient's treatment without taking into account the temporal variation of functions and behaviors. The aim of this study was to analyze the influence of the chronotype (morning type - evening type), sleep-wake state (sleep quality and excessive sleepiness) and lifestyle regularity in determining the preferred time of day for physical and mental activities following stroke. Participants responded to the MorningnessEveningness Questionnaire (MEQ), the Pittsburgh Sleep Quality Index (PSQI), the Epworth Sleepiness Scale (ESS) and the Social Rhythm Metric (SRM). The subjects were asked at what time of day they preferred to perform physical (exercises) and mental (tasks of reasoning) activities, considering only their well-being. A total of 42 patients (61 \pm 9 years) in the chronic stage after stroke ( $18 \pm 21$ months) and 12 healthy individuals took part in the study. The data were analyzed using the $\chi^{2}$ test and multiple regression. Among the patients evaluated, $64 \%$ had poor sleep quality, $43 \%$ excessive sleepiness and $57 \%$ an irregular daily routine. Regression analysis showed that chronotype was the only factor studied that influenced the preferred time of day for the activities. The results indicate the need for the physical therapist to analyze how chronotype can affect patient performance before establishing time of day of the physical therapy sessions.

Kerwords: stroke; sleep disorders; life style; circadian rhythm. 


\section{INTRODUCÃO}

Nos serviços de Fisioterapia neurológica há uma grande demanda de pacientes com acidente vascular encefálico (AVE), um déficit neurológico decorrente da restrição na irrigação sanguínea ao cérebro . Durante a avaliação fisioterapêutica, os exames da força muscular, dos reflexos, da sensibilidade e da função cognitiva são considerados fundamentais para o estabelecimento do programa de tratamento desses pacientes. No entanto, na prática clínica, o fisioterapeuta frequentemente avalia e programa o tratamento do paciente apenas em um horário, no turno da manhã, tarde ou noite, sem levar em consideração a variação temporal de funções e comportamentos, conforme abordado na Cronobiologia.

A Cronobiologia é uma área científica que estuda os ritmos biológicos mostrando variações de acordo com a hora do dia, em decorrência da regulação endógena do sistema circadiano, que é in fluenciado por pistas fóticas (alternância de claro e escuro no ambiente) e pistas não fóticas, dentre estas os horários de alimentação, atividade física e interações sociais. A principal estrutura desse sistema é o núcleo supraquiasmático (NSQ), localizado no hipotálamo e considerado como o relógio biológico, que através de conexões com a retina recebe informações sobre a luminosidade do ambiente, sincronizando os ritmos circadianos (cerca de 1 dia/24 h) com o ciclo claroescuro $^{2}$. Diferentes funções avaliadas na Cronobiologia podem ser relevantes no desempenho motor e cognitivo do indivíduo ao longo do dia, entre elas estão: o cronotipo, o padrão vigília-sono e a regularidade do estilo de vida, que por sua vez expressam a ordem temporal interna, ou seja, a coordenação dos processos orgânicos, sendo considerado um requisito para a normalidade funcional do organismo humano ${ }^{3-6}$ (Figura 1).

Em relação ao cronotipo, os indivíduos podem ser classificados em matutinos, que são os que preferem acordar e dormir cedo e apresentam bom nível de alerta e desempenho nas atividades durante a manhã; vespertinos que tendem a acordar e dormir tarde, apresentando melhor desempenho nas

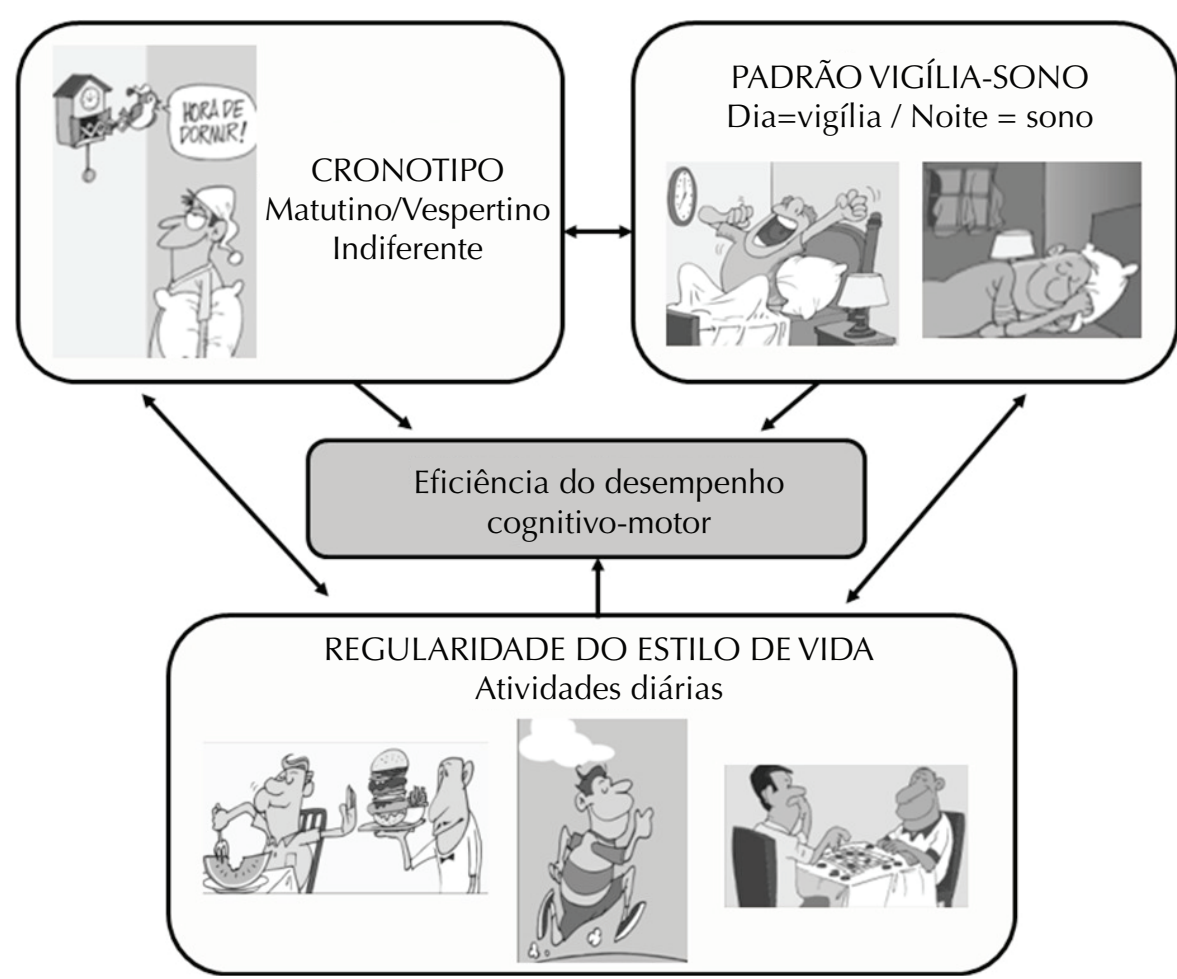

Figura 1. Modelo esquemático representando diferentes funções avaliadas na Cronobiologia: o cronotipo, o padrão vigília-sono e a regularidade do estilo de vida Essas funções bem coordenadas contribuem para maior eficência do desempenho cognitivo-motor e bem-estar do organismo humano atividades durante a tarde ou à noite; e indiferentes que são os que não têm preferência específica. O cronotipo pode ser determinado ao questionar o próprio indivíduo sobre hábitos preferidos durante as $24 \mathrm{~h}$ e registrado no Questionário de Matutinidade/ Vespertinidade $(\mathrm{QMV})^{7}$. Esse instrumento pode ser acessado, respondido e analisado através do link www.crono. icb.usp.br/cloks.htm. Quanto ao padrão vigília-sono, este compreende uma etapa de vigília, que em humanos coincide com a fase de claro e uma etapa de sono que se dá na fase de escuro. A qualidade do sono e a sonolência diurna são as principais funções analisadas ${ }^{8}$. A sonolência diurna excessiva é definida como um aumento da tendência a cochilar ou adormecer em horários ou situações inadequadas ${ }^{9,10}$. A regularidade do estilo de vida é alcançada quando os horários das atividades da vida diária tais como: levantar da cama, tomar café, almoçar, trabalhar, entre outras, são regulares, ou seja, são realizadas numa frequência de três ou mais vezes por semana em horários muito semeIhantes, expressando um ritmo social que influencia o sistema circadiano do indivíduo que é medido através do Social Rhythm Metric (SRM) ${ }^{11}$.

Apesar dos dados da literatura, ainda não se sabe qual instrumento precisa ser utilizado na avaliação dos pacientes com AVE, para determinar o melhor horário de atendimento na Fisioterapia. Nesse sentido, o objetivo do estudo foi avaliar a influência de fatores cronobiológicos na determinação do horário de preferência para a prática das atividades física e mental (raciocínio e memória, por exemplo).

METODOLOGIA

Participantes

O estudo realizado foi do tipo transversal, no qual participaram 42 pacientes diagnosticados com AVE unilateral, 20 homens e 22 mulheres, com idade média de 61 anos $( \pm 9,2)$, tempo médio de lesão de 18 meses $( \pm 21)$ e um grupo de 12 indivíduos saudáveis, composto por 6 homens e 6 mulheres, com idade média de 53 anos $( \pm 6,3)$. A amostragem foi realizada por conveniência, na qual 
os pacientes do Serviço de Fisioterapia do Hospital Universitário Onofre Lopes da Universidade Federal do Rio Grande do Norte (UFRN), que se enquadravam nos critérios de seleção adotados foram contatados e convidados a fazerem parte da pesquisa. Também através de convite foram recrutados funcionários de uma instituição de ensino superior para fazerem parte do grupo de saudáveis. Os critérios de inclusão dos participantes foram: faixa etária de 45 a 70 anos, para que não fossem incluídos indivíduos muito idosos e comprometessem o desempenho na aplicação dos instrumentos da pesquisa; ambos os sexos e indivíduos escolarizados que compreendessem todas as instruções durante a aplicação dos instrumentos. Os critérios de exclusão dos pacientes foram: lesão cerebral bilateral e recorrente, porque vários episódios de AVE poderiam confundir os resultados da avaliação; transtornos cognitivos graves, pois os pacientes não compreenderiam as instruções dos instrumentos; estágio agudo do AVE, porque poderia mascarar os resultados da avaliação; uso de antidepressivos ou neurolépticos que interferissem no padrão vigília-sono. Para os saudáveis foram: qualquer evidência clínica de patologia; uso de medicamentos que afetassem o padrão vigília-sono, trabalhador noturno que apresentasse alteração dos ritmos biológicos, assim como, pessoas que viajaram recentemente ou tiveram uma grande mudança na rotina diária.

\section{Procedimentos}

O estudo foi aprovado pelo Comitê de Ética em Pesquisa da UFRN, segundo o Parecer no 164/06, seguindo os padrões éticos de acordo com a Resolução 196/96 do Conselho Nacional de Saúde. Os participantes foram informados sobre os procedimentos da pesquisa e assinaram o termo de consentimento livre e esclarecido. Os pacientes foram submetidos a uma avaliação clínica e o diagnóstico foi estabelecido através de tomografia computadorizada. Os indivíduos saudáveis foram avaliados através do Cumulative IIIness Rating Scale (CIRS) a fim de excluir qualquer patologia.

Durante uma semana, os indivíduos preencheram o SRM em suas residências, registrando a cada dia a hora em que realizavam 17 atividades: levantar da cama, primeiro contato com uma pessoa, tomar bebida, tomar café da manhã, sair de casa, começar a trabalhar, almoçar, cochilar, jantar, fazer exercícios, fazer um lanche, assistir a programas de notícias à noite, assistir a outros programas de televisão, voltar para casa, ir para a cama, além de duas outras atividades que eles realizassem regularmente durante a semana. Os escores do SRM variam de 0 e 7 (de menor a maior regularidade).

Em seguida, foram aplicados os seguintes questionários: o QMV, no qual os escores entre 16 e 41 determinam o tipo vespertino, entre 59 e 86 define o tipo matutino e entre 42 e 58 o tipo indiferente; o IQSP com escores variando de 0 a 21, no qual escores maiores do que 5 implica em uma qualidade ruim do sono; e o questionário de Sonolência Excessiva de Epworth (SEE), que avalia a chance de cochilar em 8 situações apresentadas, pontuando de 0 (nenhuma chance) a 3 (grande chance). O escore final varia de 0 a 24, com pontuações iguais ou maiores que 10 determinando sonolência diurna excessiva. Por fim, foi questionado aos participantes em qual horário eles preferiam realizar atividade física (exercícios) e mental (tarefas de raciocínio e memória, por exemplo), se pela manhã ou a tarde. Aos pacientes foram dadas explicações para que considerasse apenas seu bem-estar pessoal, sem influência de horários do serviço de Fisioterapia, de transporte, disponibilidade do acompanhante, de trabalho e estudo.

\section{Análise estatística}

Para a análise dos dados foi utilizado o programa SPSS 12.0, atribuindo-se o nível de significância de 5\%. Foi realizado o teste de normalidade KolmogorovSmirnov para as variáveis quantitativas. Diferenças entre pacientes e saudáveis quanto ao cronotipo, qualidade do sono, sonolência e à regularidade do estilo de vida foram analisadas através do teste $\chi^{2}$ (correção de Yates) para variáveis categóricas e o Teste $t$ de Student de amostras independentes para variáveis contínuas. Modelo explicativo foi construído através da análise de regressão linear múltipla, a fim de identificar as variáveis preditoras do horário preferencial de prática das atividades física e mental.
RESULTADOS

Pela análise realizada do cronotipo, 39 pacientes foram classificados como matutinos e 3 como indiferentes, e todos os indivíduos saudáveis foram classificados como matutinos, não havendo nenhum indivíduo vespertino na amostra estudada. Não foram verificadas diferenças significativas entre os grupos quanto às médias do cronotipo $(p=0,969)$.

Os resultados mostraram que $64 \%$ dos pacientes e $42 \%$ dos saudáveis apresentaram qualidade ruim do sono, não sendo essa diferença significativa, assim como, para as médias encontradas $(p=0,253)$. Na avaliação da sonolência, $43 \%$ dos pacientes e $42 \%$ dos saudáveis apresentaram sonolência excessiva, mas essa diferença não foi estatisticamente significativa, da mesma forma entre as médias dos grupos ( $p=0,388)$. Na avaliação da regularidade da rotina diária, não foi observada diferença significativa entre os grupos quanto à média do SRM ( $p=0,187)$, apesar de $57 \%$ dos pacientes e $83 \%$ dos saudáveis terem apresentado padrão irregular da rotina diária (Tabela 1).

A média do horário preferencial para a prática de atividade física foi de $10 \mathrm{~h} 45$ ( $\pm 21 \mathrm{~min}$.) para pacientes e 9h50 ( \pm 7 min.) para os saudáveis, não havendo diferença significativa entre os grupos $(p=0,371)$. Para a atividade mental, a média do horário preferencial foi às $12 \mathrm{~h} 31$ ( $\pm 1 \mathrm{~min}$.) para pacientes e $11 \mathrm{~h} 00$ ( \pm 8 min) para saudáveis, também não encontrada diferença significativa entre os grupos $(p=0,233)$.

Ao final da análise de regressão verificou-se que as variáveis: qualidade do sono, sonolência diurna e regularidade do estilo de vida não contribuíram significativamente para o horário preferencial de prática das atividades física e mental, e o cronotipo foi o único fator que teve influência significativa para ambos os horários (Tabela 2).

\section{DISCUSSÃO}

Os resultados encontrados não mostraram diferenças entre os grupos (pacientes e saudáveis) em todos os instrumentos avaliados, em função de que ambos os grupos apresentavam semelhanças 
Tabela 1. Comparação entre pacientes e indivíduos saudáveis quanto à média \pm desvio padrão (DP) do cronotipo, da qualidade do sono, da sonolência excessiva e da regularidade do estilo de vida

\begin{tabular}{lccc}
\hline VARIÁVEIS & PACIENTES & SAUDÁVEIS & \\
\hline & Média \pm DP & Média \pm DP & Valor $p$ \\
\cline { 2 - 4 } Cronotipo (QMV) & $67,9 \pm 3,6$ & $68,0 \pm 7,2$ & 0,969 \\
Qualidade do sono (IQSP) & $4,9 \pm 1,8$ & $6,1 \pm 3,3$ & 0,254 \\
Sonolência excessiva (SEE) & $11,0 \pm 5,6$ & $9,6 \pm 4,5$ & 0,388 \\
Regularidade do estilo de vida & $4,7 \pm 1,1$ & $5,1 \pm 0,8$ & 0,187 \\
(SRM) & &
\end{tabular}

QMV: Questionário de Matutinidade/Vespertinidade; IQSP: Índice de Qualidade do Sono de Pittsburgh; SEE: Sonolência Excessiva de Epworth; SRM: Social Rhythm Metric

Tabela 2. Modelos de regressão linear múltipla, considerando o cronotipo, qualidade do sono e sonolência excessiva e a regularidade do estilo de vida, como variáveis independentes e a hora preferencial de prática das atividades física e mental, como variáveis dependentes

\begin{tabular}{lccc}
\hline & $\beta \pm E$ Erro padrão & $\begin{array}{c}\text { Intervalo de } \\
\text { confiança (95\%) }\end{array}$ & Valor p \\
\hline Modelo - Física & & & \\
$\quad$ Cronotipo & $-0,010 \pm 0,004$ & $-0,017-0,003$ & 0,005 \\
$\quad$ Qualidade do sono & $0,044 \pm 0,043$ & $-0,043-0,131$ & 0,318 \\
$\quad$ Sonolência & $-0,007 \pm 0,045$ & $-0,098-0,085$ & 0,886 \\
$\quad$ Regularidade do estilo de vida & $0,002 \pm 0,028$ & $-0,055-0,055$ & 0,950 \\
Modelo - Mental & & & \\
$\quad$ Cronotipo & $-0,007 \pm 0,003$ & $-0,012-0,001$ & 0,020 \\
$\quad$ Qualidade do sono & $0,042 \pm 0,035$ & $-0,028-0,111$ & 0,235 \\
$\quad$ Sonolência & $-0,033 \pm 0,036$ & $-0,106-0,040$ & 0,368 \\
Regularidade do estilo de vida & $-0,022 \pm 0,023$ & $-0,068-0,024$ & 0,337 \\
\hline
\end{tabular}

Modelo - Física: $r=0,32 ; r^{2}=0,10 ; r^{2}$ ajustado=0,08; Modelo - Mental: $r=0,21$; $r^{2}=0,04 ; r^{2}$ ajustado $=0,02$

quanto ao caráter de matutinidade/ vespertinidade e também porque apresentavam alterações importantes em relação ao padrão de sono-vigília e à regularidade da rotina diária. Mesmo os indivíduos saudáveis não apresentaram qualquer tipo de patologia, é possível que os esquemas de trabalho e a faixa etária tenham influenciado o padrão vigíliasono e a regularidade da rotina diária, justificando também a não diferença entre os grupos.

Os pacientes avaliados mostraram um padrão predominantemente irregular de realização de atividades diárias. Considerando que muitos dos indivíduos da amostra encontravam-se no estágio crônico do AVE, esse dado pode ser justificado pelo fato de que pacientes com mais tempo de lesão poderiam estar menos expostos as pistas fóticas, uma vez que a maioria frequentemente não retorna às suas atividades sociais e ocupacionais diárias ${ }^{12}$. A maior atividade social desses pacientes poderia favorecer a exposição à luz natural, aproveitando o alto nível de luz solar encontrado em Natal (RN), cidade na qual o estudo foi realizado. Um achado importante do estudo anterior foi a correlação entre o SRM e o National Institute of Health Stroke Scale (NIHSS), confirmada pela associação entre os pacientes com menos regularidade e mais dano neurológico. Neste caso, o SRM pode ser considerado um preditor de dano neurológico em pacientes com $\mathrm{AVE}^{13}$.

Quanto à qualidade do sono e a sonolência diurna, verificamos que dos pacientes avaliados, 64\% apresentaram qualidade ruim do sono e $43 \%$ sonolência excessiva. De acordo com a literatura, pacientes com um único evento isquêmico mostraram uma correlação entre o desempenho da memória verbal e não verbal com a quantidade de sono de ondas lentas, sono REM e eficiência do sono, sugerindo uma ligação entre o sono e as funções cognitivas após o AVE ${ }^{14,15}$. Assim, a investigação de problemas relacionados ao sono é um importante componente da abordagem diagnóstica e terapêutica clínica.

No presente estudo, as médias dos horários escolhidos para a prática das atividades estavam compreendidas predominantemente no turno da manhã, o que corresponde ao fato de que a amostra possuía mais indivíduos matutinos, o que foi confirmado pela análise de regressão. Ao avaliar a influência das funções cronobiológicas na preferência dos pacientes quanto ao horário de prática das atividades física e mental, não foi encontrada correlação com a regularidade da rotina diária, a qualidade do sono e a sonolência com o horário preferencial de realização das atividades, mostrando que esses fatores podem não ter influência específica na escolha do horário de atendimento fisioterapêutico na amostra estudada.

Entretanto, o cronotipo foi o único fator que influenciou o horário preferencial, tanto para a prática de atividade física, quanto mental, indicando que quanto maior o valor do cronotipo (mais matutino) menor o valor da hora (mais cedo) escolhido para realizar essas atividades. Dados da literatura mostram que o cronotipo é um dos critérios mais fortes para discriminar diferenças individuais no ritmo circadiano de variáveis fisiológicas e na avaliação do alerta ${ }^{16,17}$. Os estudos indicam que indivíduos matutinos e vespertinos, em geral, apresentam maior rendimento em tarefas quando as realizam no horário correspondente ao seu cronotipo. A literatura especifica esses achados como um "efeito de sincronia", no qual o desempenho cognitivo-motor é melhor quando os testes são realizados no momento "ótimo" e cai substancialmente na hora "não ótima"18,19. Esses dados dão mais fundamento à importância de avaliar o cronotipo dos pacientes antes do planejamento do tratamento fisioterapêutico, pois os profissionais correm o risco de subestimar ou superestimar o desempenho motor e cognitivo dependendo do horário de realização dos testes na prática clínica. É possível 
que o tratamento sendo realizado no horário em que o paciente se encontra mais apto ao desenvolvimento de atividades, seu processo de recuperação seja otimizado.

Os dados encontrados no presente estudo também reforçam a importância de se identificar os instrumentos necessários para uma avaliação adequada dos pacientes com $\mathrm{AVE}^{20}$, principalmente na área da Cronobiologia. Além disso, esses resultados podem dar embasamento para as novas terapias propostas na reabilitação do AVE, tais como a imagética motora ${ }^{21}$ e a prática observacional ${ }^{22}$ que demandam elevado processamento cognitivo em associação com a função motora, que por sua vez podem ser influenciadas pelo cronotipo de cada paciente.

O estudo realizado apresentou algumas limitações, tais como, a falta de

avaliações objetivas do padrão sonovigília pela polissonografia e da regularidade das atividades diárias pela actimetria, que explicassem melhor os comprometimentos apresentados; assim como não foi avaliada nesse estudo a relação entre os problemas de sono e a localização das lesões cerebrais, em função dos exames de neuroimagem terem sido realizados em diferentes hospitais, não permitindo a padronização dos laudos médicos. No entanto, os resultados encontrados têm grande importância para a amostra estudada, pois expressam algumas alterações do sono, cognitivas e funcionais que devem ser abordadas através de conhecimentos da Cronobiologia.

CONCLUSÃO

O estudo realizado conseguiu evidenciar uma associação entre o cronotipo e o horário de preferência para a prática das atividades mental e física em pacientes pós-AVE com as características da população da qual a amostra foi extraída, sugerindo ser esse um instrumento de avaliação importante para se estabelecer o horário de atendimento na Fisioterapia. Há necessidade de que os conceitos da Cronobiologia sejam compreendidos e largamente utilizados em função da saúde de seus pacientes. Pretende-se dessa forma, com esse estudo incentivar os fisioterapeutas a realizarem pesquisas em Cronobiologia, visto ser este um profissional que tem um amplo objetivo de proteger e restaurar a identidade pessoal e social do indivíduo.

\section{AGRADECIMENTOS}

Ao chargista Ivan Kabral pela confecção dos desenhos.

\section{REFERÊNCIAS}

1. World Health Organization (WHO). WHO STEPS Stroke Manual: The WHO STEPwise approach to stroke surveillance. Geneva: World Health Organization; 2006.

2. Schulz P, Steimer T. Neurobiology of circadian systems. CNS Drugs. 2009;23(Suppl 2):3-13.

3. Rana S, Mahmood S. Circadian rhythm and its role in malignancy. J Circadian Rhythms. 2010;8:1-13.

4. Lund HG, Reider BD, Whiting AB, Prichard JR. Sleep patterns and predictors of disturbed sleep in a large population of college students. J Adolesc Health. 2010;46(2):124-32.

5. Monk TH. Enhancing circadian zeitgebers. Sleep. 2010;33(4):421-2.

6. Zisberg A, Gur-Yaish N, Shochat T. Contribution of routine to sleep quality in community elderly. Sleep. 2010;33(4):509-14.

7. Horne JA, Östberg O. A self-assessment questionnaire to determine morningness-eveningness, in human circadian rhythms. Int J Chronobiol. 1976;4(2):97-110.

8. Buysse DJ, Reynolds CF 3rd, Monk TH, Hoch CC, Yeager AL, Kupfer DJ. Quantification of subjective sleep quality in healthy elderly men and women using the Pittsburgh Sleep Quality Index (PSQI). Sleep. 1991;14(4):331-8.

9. Johns MW. A new method for measuring daytime sleepiness: the Epworth sleepiness scale. Sleep. 1991;14(6):540-5.
10. Lovato N, Lack L. The effects of napping on cognitive functioning. Prog Brain Res. 2010;185:155-66.

11. Monk TH, Flaherty JF, Frank E, Hoskinson K, Kupfer DJ. The Social Rhythm Metric: an instrument to quantify the daily rhythms of life. J Nerv Ment Dis. 1990;178(2):120-6.

12. Carod-Artal FJ, Egido JA. Quality of life after stroke: the importance of a good recovery. Cerebrovasc Dis. 2009;27(Suppl 1):204-14.

13. Campos TF, Silveira ABG, Barroso MTM. Regularity of daily activities in stroke. Chronobiol Int. 2008;25(4):611-24.

14. Jackson ML, Howard ME, Barnes M. Cognition and daytime functioning in sleep-related breathing disorders. Prog Brain Res. 2011;190:53-68.

15. Siccoli MM, Rölli-Baumeler N, Achermann P, Bassetti CL. Correlation between sleep and cognitive functions after hemispheric ischaemic stroke. Eur. J. Neurol. 2008;15:(6) 565-72.

16. Korczak AL, Martynhak BJ, Pedrazzoli M, Brito AF, Louzada FM. Influence of chronotype and social zeitgebers on sleep/wake patterns. Braz J Med Biol Res. 2008;41(10):914-9.

17. Roepke SE, Duffy JF. Differential impact of chronotype on weekday and weekend sleep timing and duration. Nat Sci Sleep. 2010;2010(2):213-20. 


\section{Referências (cont.)}

18. Rowe G, Hasher L, Turcotte T. Age and synchrony effects in visuospatial working memory. Q J Exp Psychol (Colchester). 2009;62(10):1873-80.

19. Matchock RL, Mordkoff JT. Chronotype and timeof-day influences on the alerting, orienting, and executive components of attention. Exp Brain Res. 2009;192(2):189-98.

20. Faria CDCM, Saliba VA, Teixeira-Salmela LF, Nadeau S. Comparação entre indivíduos hemiparéticos com e sem histórico de quedas com base nos componentes da Classificação Internacional de Funcionalidade, Incapacidade e Saúde. Fisioter Pesq. 2010;17(3): 242-47.

21. Trevisan CM, Trintinaglia V. Efeito das terapias associadas de imagem motora e de movimento induzido por restrição na hemiparesia crônica: estudo de caso. Fisioter Pesq. 2010;17(3):264-69.

22. Celnik P, Webster B, Glasser DM, Cohen LG. Effects of action observation on physical training after stroke. Stroke. 2008;39(6):1814-20. 